\title{
Management of babies born extremely preterm at less than 26 weeks of gestation: a framework for clinical practice at the time of birth
}

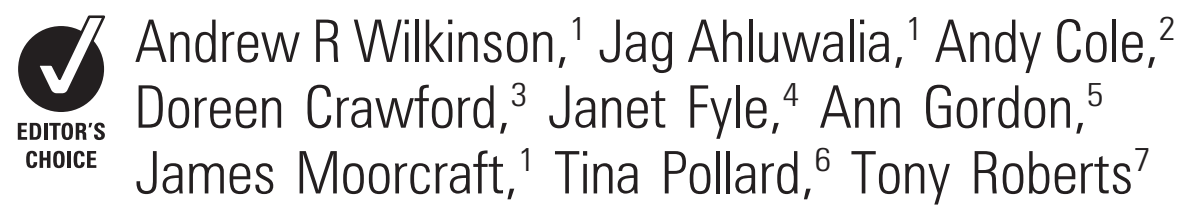

Management of the delivery of an extremely preterm baby is one of the most challenging aspects of perinatal medicine. The ethical, social, economic and legal issues have recently been reviewed by the Nuffield Council on Bioethics. ${ }^{1}$ The professions and advocates for parents were encouraged to consider the pattern of care appropriate for babies born before 26 weeks' gestation on the basis of the best information currently available. There are limitations to contemporary evidence, particularly in terms of predicting outcome after the shortest of pregnancies. While recognising these limitations, and although every pregnancy is different, some general principles can be described.

This is not a set of instructions, but a framework to highlight the range of evidence and opinion that needs to be considered by staff and parents. Care of the mother, her fetus and the baby will always need to be individualised and should be led by senior staff in all disciplines. The parents' hopes and expectations need to be explored with honesty and compassion in a realistic way, drawing upon the available evidence. Communication and agreed plans must be documented in full and signed legibly. These plans may need to be revised frequently.

\footnotetext{
${ }^{1}$ BAPM, London, UK; ${ }^{2}$ BLISS, London, UK; ${ }^{3}$ RCN, London, UK; ${ }^{4} \mathrm{RCM}$, London, UK; ${ }^{5} \mathrm{RCPCH}$, London, UK; ${ }^{6} \mathrm{NNA}$, London, UK; ${ }^{7} \mathrm{RCOG}$, London, UK

Correspondence to: Professor Andrew R Wilkinson, University of Oxford, Department of Paediatrics, Neonatal Unit, Women's Centre, John Radcliffe Hospital, Oxford OX3 9DU, UK; andrew.wilkinson@paediatrics.ox.ac.uk

Report of a Working Group of the British Association of Perinatal Medicine
}

\section{BEFORE DELIVERY}

When it appears that a mother will deliver her baby at a very early gestational age, there is important clinical information that needs to be carefully reviewed. Accurate information will greatly assist the dialogue and inform the decisions made. Whenever possible antenatal management decisions should involve both of the parents and the clinical staff who will be responsible before and after the delivery.

The obstetric history and antenatal care must be considered carefully, with particular attention to the ultrasound dating scan(s). The earlier this has been carried out the more accurately the gestational age will be known. ${ }^{2}$ Other information about fetal growth or abnormalities may be available from the scan(s). The best estimation of gestational age should be agreed with the parents. A record of the discussion must be made and revised according to any changes in condition of the fetus or mother.

Discussion with the parents must include information about the expected outcome based on the best available local and national population data. Care must be taken in interpreting local hospital statistics which, at very low gestational ages, will be based on small numbers even in the largest centres.

The practicalities of starting, withholding and withdrawing intensive care and the positive role of palliative care where appropriate should be described to the parents. This will help to prepare them for the different possible outcomes after delivery.

Parents may find the advice and support of their family, friends and spiritual advisers to be of great value at this time.

Assessment of the local neonatal unit staffing and capabilities must be made.
Transfer to another hospital, increasingly likely within a managed clinical network, should be discussed if this is clinically appropriate. Written information that includes this possibility should be given to all parents at the time of booking.

If time allows, the parents should be offered the opportunity to visit the neonatal unit.

\section{MANAGEMENT RECOMMENDATIONS}

On the basis of the best assessment of gestational age, as well as information about the well-being of the fetus and the wishes of the parents, a clear plan for delivery and care of the baby must be made and documented. This will need to be reviewed regularly (fig 1).

Discussion about the mode of delivery should include an explanation of the maternal morbidity in future pregnancies associated with a classical caesarean section when carried out at very early gestational age. However, this may be necessary for maternal indications.

If active obstetric intervention in the interests of the fetus is not planned, continuous monitoring of the fetal heart rate is not advised. However, the parents should be made aware that their baby may show signs of life for a variable time after birth, and intermittent assessment of the fetus by a Doppler device or auscultation is useful to the professionals responsible at the time of birth.

\section{RESUSCITATION AT BIRTH}

Preterm labour often progresses rapidly. In these circumstances, there may be insufficient time to hold a detailed discussion with the parents before the baby is born. A decision about resuscitation may need to be made on the basis of the most recent management plan, if any, and the available clinical information. When lung inflation with a mask is an appropriate initial approach, this should be carried out as described in the Newborn Life Support course handbook. ${ }^{3}$

\section{A FRAMEWORK FOR CLINICAL PRACTICE}

This was developed on the basis of consensus and the most recent evidence available.

\section{(A) Less than $23^{+0}$ weeks}

If gestational age is certain and less than $23^{+0}$ (ie, 22 weeks), it would be considered in the best interests of the baby, and standard practice, for resuscitation not to be carried out. If the parents wish, they should have the opportunity to discuss 
Box 1 Factors to be taken into account when discussing management with parents

Antenatal factors influencing fetal outcome

- Gestational age

- Steroid administration

- Predicted fetal weight

- Multiple pregnancy

- Sex

- Presence and severity of pathology

- Fetal growth restriction

- Fetal acidaemia (as suggested by an abnormal cardiotocograph or umbilical artery Doppler flow velocity waveform (particularly absent or reversed end diastolic frequencies))

- Sepsis

- Fetal anomaly

Parental factors

- Cultural

- Religious

- Medical

- Past obstetric history

- Previous pregnancy loss

- Sub-fertility

Parental expectations

- Understanding of process

- In utero transfers

- Postnatal assessment

- Paediatric involvement/interventions

- Outcome

- Survival

- Morbidity

- Their wishes

Condition of infant at delivery

- Apparent maturity

- Extensive bruising

- Heart rate

- Spontaneous activity level

- Respiratory effort and signs of sustained response to resuscitation

outcomes with a second senior member of the perinatal team.

In the EPICure study of all babies born in 1995 in the UK and Eire at <26 weeks' gestational age, only two babies reported on at $<23$ weeks survived to discharge and one has severe disability (table 1). ${ }^{4}$

In the EPICure 2 study (2006), survival remains extremely rare at this gestational age, with a high incidence of early major morbidity in the few who are discharged home. $^{7}$

\section{(B) $23^{+0}-23^{+6}$ weeks}

If gestational age is certain at $23^{+0}-23^{+6}$ (ie, 23 weeks) and the fetal heart is heard during labour, a professional experienced in resuscitation should be available to attend the birth. In the best interests of the baby, a decision not to start resuscitation is an appropriate approach, particularly if the parents have expressed this wish. However, if resuscitation is started with lung inflation using a mask, the response of the heart rate will be critical in deciding whether to continue or to stop and sensitively explain to the parents the futility of further interventions.

The EPICure study (1995) reported in 2000 that, at 23 weeks, 121/241 (50\%) of live born babies were admitted for intensive care, of whom 105 (80\%) died in hospital. ${ }^{4}$ Twenty-six babies were discharged home, one died and 14 (54\%) have a moderate or severe disability at 6 years of age (table 1$){ }^{5}$

Early findings in the EPICure 2 study (2006) show that, at this gestational age, survival has not increased significantly and there has been no change in early major morbidity.

\section{(C) $24^{+0}-24^{+6}$ weeks}

If gestational age is certain at $24^{+0}-$ $24^{+6}$ weeks, resuscitation should be started unless the parents and clinicians have considered that the baby will be born severely compromised. However, the response of the heart rate to lung inflation using a mask will be critical in deciding whether to proceed to intensive care. If the baby is assessed to be more immature than expected, deciding not to start resuscitation may be considered in the best interest of the baby.

In the 1995 study, ${ }^{4}$ although 313/382 (78\%) of babies born at this gestational age (313) were given intensive care, 198 $(66 \%)$ died. ${ }^{3}$ Half of the survivors (52) have a moderate or severe disability at 6 years of age (table 1$){ }^{6}$

Early findings in the EPICure 2 study (2006) show that, at this gestational age, survival has increased significantly by $12 \%$. More babies were treated for retinopathy of prematurity, but there is no evidence of any change in other early major morbidity. ${ }^{7}$

\section{(D) $\mathbf{2 5}$ weeks and greater}

When gestational age is $25^{+0}$ weeks or more, survival is now considerably greater than in 1995. It is appropriate to resuscitate babies at this gestation and, if the response is encouraging, to start intensive care.

In the 1995 study, ${ }^{4} 389 / 424$ (92\%) babies born alive at 25 weeks were admitted for intensive care, but 171 (48\%) died. ${ }^{3}$ Of the survivors, $27 \%$ had no identifiable impairment at 6 years of age. ${ }^{6}$

In 2006, survival had increased significantly from $54 \%$ to $67 \%$, but there is no evidence of any change in early major morbidity. $^{5}$

\section{(E) Uncertain gestational age}

If gestational age is uncertain (ie, no dating ultrasound scan) but thought to be $\geqslant 23^{+0}$ weeks, an ultrasound scan by an experienced sonographer should be carried out if time permits. If the fetal heart is heard during labour, a professional experienced in resuscitation and another clinician (neonatal nurse or trainee paediatrician) should be called to attend the birth. A decision should then be made, in the best interests of the baby, as to whether resuscitation should begin with mask ventilation. Once begun, the response of the heart rate to lung inflation will be crucial in judging how long to continue resuscitation. If there is any uncertainty about management, guidance from more senior staff should be sought urgently. 


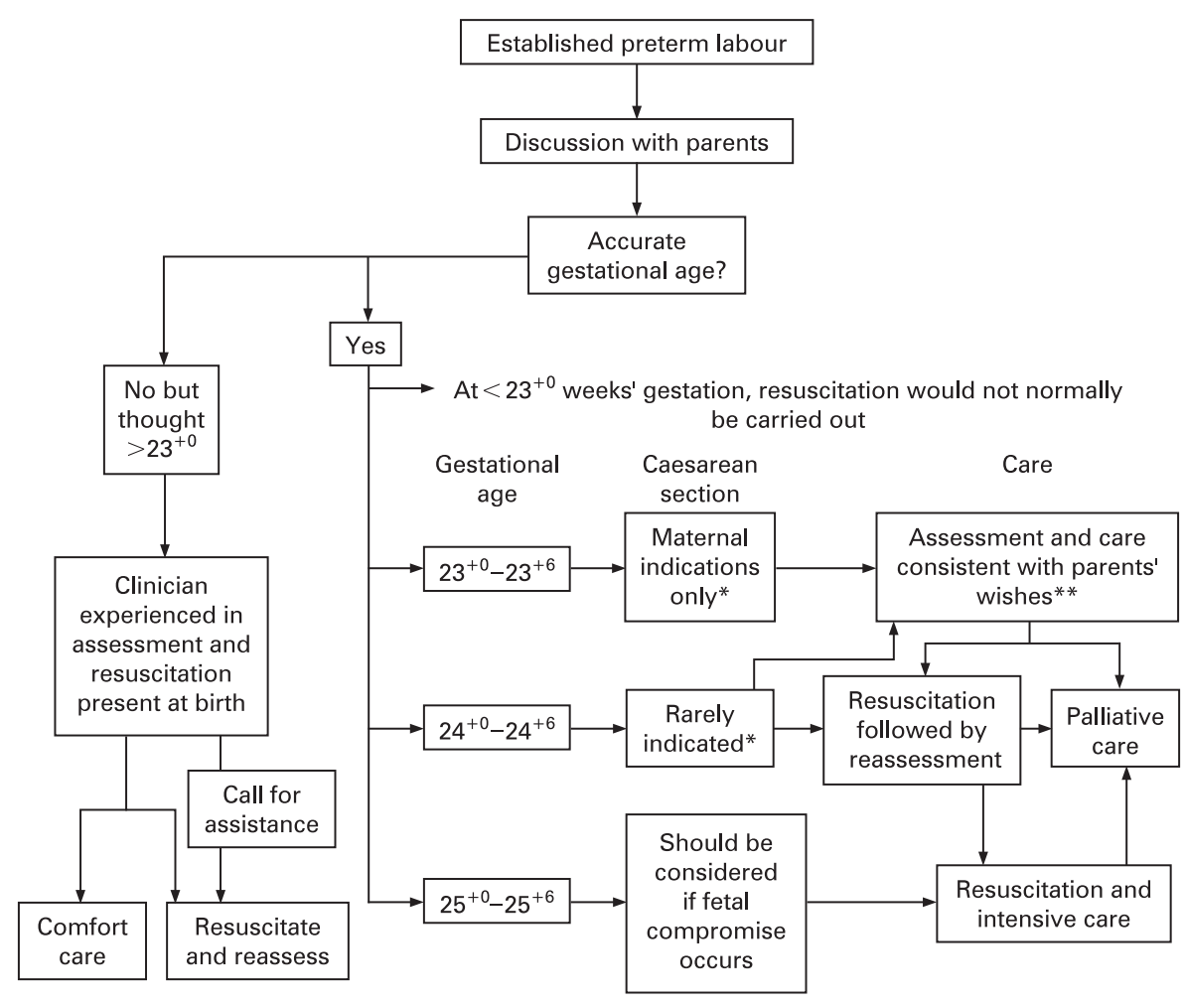

Figure 1 Management of babies born extremely preterm at $<26$ weeks' gestation. ${ }^{*}$ Caesarean section offers no benefit to the fetus $<25$ weeks' gestation and should be performed only when indicated for the health of the mother. ${ }^{* *}$ Survival and outcome for infants born at $23^{+0}-24^{+6}$ is poor. Management of an infant born at this gestation should be consistent with parents' wishes, but decisions made before birth are influenced by the baby's condition at birth. When parents wish resuscitation, the clinician's decision to resuscitate should depend on detailed assessment of the infant's condition. Objective criteria include movements, lack of bruising, presence of spontaneous respiratory efforts, and response to initial resuscitation.

Table 1 Summary of outcomes for all live births $<26^{+0}$ weeks' gestation in UK and Ireland, March to December $1995^{3}$

\begin{tabular}{|c|c|c|c|c|}
\hline \multirow[b]{2}{*}{ Outcome } & \multicolumn{4}{|c|}{ N (\% live births) } \\
\hline & 22 weeks & 23 weeks & 24 weeks & 25 weeks \\
\hline *Live births & 138 & 241 & 382 & 424 \\
\hline Died in delivery room & $116(84)$ & $110(46)$ & $84(22)$ & $67(16)$ \\
\hline Admitted for intensive care & $17(12)$ & $121(50)$ & $313(82)$ & 389 (92) \\
\hline Survival & $2(1.5)$ & $26(11)$ & $100(26)$ & $186(44)$ \\
\hline$\dagger$ Gestational age reassessed ${ }^{4}$ & $22(16)$ & $131(54)$ & $298(78)$ & $357(84)$ \\
\hline Died in NICU (\% admitted) & $20(91)$ & $105(80)$ & $198(66)$ & $171(48)$ \\
\hline Survived to discharge & $2(9)$ & $26(20)$ & $100(33)$ & $186(52)$ \\
\hline Deaths after discharge (\% of discharged babies) & 0 & $1(4)$ & $2(2)$ & $3(1.6)$ \\
\hline Lost to follow-up & 0 & 3 & 25 & 39 \\
\hline † Severe disability & $1(50)$ & $5(23)$ & $21(28)$ & $40(18)$ \\
\hline Moderate or mild disability & 1 & $14(61)$ & $42(56)$ & $83(56)$ \\
\hline Survivors without identified impairment at 6 years ${ }^{4}$ & 0 & $3(1)$ & $10(3)$ & $35(8)$ \\
\hline $\begin{array}{l}\text { Survivors without identified disability at } 6 \text { years (\% } \\
\text { admissions) }{ }^{4}\end{array}$ & 0 & $3(2)$ & $10(4)$ & $35(11)$ \\
\hline
\end{tabular}

Further early outcome data from the EPICure 2 study of babies born $<27$ weeks' gestational age in England in 2006 will be available later in 2008.

*Gestational age based on "working" estimate on labour ward.

†Gestational age assessed postnatally.

$\downarrow$ A severe disability is defined as one that is expected to render the child dependent. ${ }^{4}$

\section{INTENSIVE CARE}

The response of the baby to mask ventilation is critical in deciding whether to start intensive care. If the heart rate increases rapidly and the colour improves, appropriate ventilatory support, including intubation and surfactant therapy, should be given, and the baby transferred to the neonatal unit for further assessment.

There is no evidence to support the use of epinephrine by any route, or chest compressions, during resuscitation at gestational age $<26$ weeks. ${ }^{8}$

Management should be decided by doctors and nurses experienced in neonatal intensive care.

\section{WITHHOLDING OR WITHDRAWING RESUSCITATION OR INTENSIVE CARE}

When resuscitation or intensive care is withheld or withdrawn, the baby should be given all the care needed for his/her comfort and the parents encouraged by appropriate staff to hold and spend time with their baby, if they wish, in a quiet and private location. Further recommendations can be found in a Royal College of Paediatrics and Child Health monograph on withholding or withdrawing life-sustaining treatment. ${ }^{9}$

When a baby dies, the parents should be offered bereavement counselling, including advice about post-mortem examination. At an appropriate time, the prognosis for future pregnancies should also be discussed.

Acknowledgements: BAPM would like to thank those members of the Working Group from other organisations who made such valuable contributions to this report. The Working Group thanks all those who commented on earlier drafts and those who responded during the consultation period, particularly all the members and executive committee of BAPM. The Group is also grateful for the additional comments made by Malcolm Chiswick, Kate Costeloe, Alison Leaf and Edile Murdoch from BAPM and Stephen Robson from RCOG.

\section{Competing interests: None.}

Endorsed by the Executive Committee of the Association.

Accepted 9 September 2008

Published Online First 6 October 2008

Arch Dis Child Fetal Neonatal Ed 2009;94:F2-F5. doi:10.1136/adc.2008.143321

\section{REFERENCES}

1. Nuffield Council on Bioethics. Critical care decisions in fetal and neonatal medicine: ethical issues. London: Nuffield Council on Bioethics, 2006. www. nuffieldbioethics.org (accessed 29 Oct 2008).

2. NICE. Evidence based clinical guideline: antenatal care routine care for the healthy pregnant woman. October 2003. 1.2.6 Gestational age assessment: LMP and ultrasound 4.6:34. http://www.rcog.org.uk/ index.asp?PagelD $=693$ (accessed 29 Oct 2008).

3. Resuscitation Council (UK). Newborn Life Support. Resuscitation at Birth. 2nd edn. 2006. www.resus.org uk (accessed 29 Oct 2008). 
4. Costeloe K, Hennessy E, Gibson AT, et al. Outcome to discharge from hospital for infants at the threshold of viability. Pediatrics 2000;106:659-71.

5. Wood NS, Marlow N, Costeloe K, et al. Neurologic and developmental disability after extremely preterm birth. $N$ Engl J Med 2000:343:378-84.
6. Marlow N, Wolke D, Bracewell M, et al. Neurologic and developemental disability at 6 years of age after extremely preterm birth. $N$ Engl J Med 2005;352:9-19.

7. EPICure 2 Perinatal Group. Survival and early morbidity of extremely preterm babies in England: changes since1995. Arch Dis Child 2008;93:(Suppl 1):A33-4.
8. Sims DG, Heal CA, Bartle SM. Use of adrenaline and atropine in neonatal resuscitation. Arch Dis Child Fetal Neonatal Ed 1994;70:F3-9.

9. Royal College of Paediatrics and Child Health Withholding or withdrawing life sustaining treatment in children - a framework for practice. 2nd edn. London: RCPCH, 2004.

\section{Single versus multiple antenatal steroids in threatened preterm delivery: more benefit or harm?}

\section{Gusztav Belteki, ${ }^{1}$ Gordon C S Smith ${ }^{2}$}

The first demonstration that antenatal maternal glucocorticoid treatment reduced neonatal morbidity was reported by Liggins and Howie in 1972. In the following two decades antenatal corticosteroid prophylaxis gradually found its way into clinical practice and had become an accepted part of the standard care by the early 1990s. ${ }^{23}$ It clearly reduces overall neonatal mortality, the risk of respiratory distress syndrome (RDS) and the need for respiratory support. ${ }^{4}$ It also decreases the risk of other complications of prematurity, including intraventricular haemorrhage (IVH) and necrotising enterocolitis but not bronchopulmonary dysplasia (BPD). The current recommendation is to give two doses of $12 \mathrm{mg}$ betamethasone, $24 \mathrm{~h}$ apart, to women who may deliver within 7 days and are less than 35 weeks pregnant..$^{5-7}$ Most benefit is seen when delivery follows the second dose by more than $24 \mathrm{~h}$; however, even an incomplete course reduces neonatal morbidity and mortality. ${ }^{8}$

There is a marked reduction in the apparent beneficial effects of antenatal steroids (ie, glucocorticoids) if the interval between administration and delivery exceeds 7 days. ${ }^{4}$ This led to the evaluation of multiple steroid treatments among women who remained at high risk of preterm birth. ${ }^{9}$ However, animal data and observational human studies demonstrated possible adverse effects of repeated doses of glucocorticoids. Moreover, evidence emerged of severe adverse effects of

\footnotetext{
${ }^{1}$ Neonatal Intensive Care Unit, Rosie Maternity Hospital, Addenbrooke's Hospital, Cambridge, UK; ${ }^{2}$ Department of Obstetrics and Gynaecology, Cambridge University, Cambridge, UK
}

Correspondence to: Dr Gordon C S Smith, Department of Obstetrics and Gynaecology, Cambridge University, Rosie Maternity Hospital, Cambridge CB2 2SW, UK; gcss2@cam.ac.uk postnatal steroids, particularly on growth and neurodevelopmental outcome. ${ }^{10}$ This resulted in guidelines advising against routine use of repeat steroid courses except in clinical studies, in both the UK and the USA. ${ }^{511}$ In this paper, we discuss the data from a series of randomised controlled trials (RCTs) of repeat steroids and the implications for care of women at sustained risk of preterm birth.

\section{BENEFICIAL EFFECTS}

The physiological consequences of repeated antenatal steroid exposure have been extensively studied in animals, mostly in sheep. In preterm lambs, an increasing number of weekly maternal steroid doses $^{1-4}$ resulted in increasing improvement in lung compliance, ventilation efficiency ${ }^{12}{ }^{13}$ and vascular function (as demonstrated by increasing endothelial nitric oxide synthase activity).$^{14}$ However, detailed histological analysis failed to demonstrate additional fetal lung maturation when compared with a single course. $^{15}$

In the last 10 years, several observational clinical studies have analysed infant outcomes in relation to repeat steroid exposure. Some of them failed to demonstrate beneficial neonatal effects, ${ }^{16}{ }^{17}$ and one of them reported an increased risk of IVH. ${ }^{18}$ The major problem of interpreting observational studies is the possibility of bias. In this case, the primary concern is that repeat steroids may be associated with adverse effects through the indication for their use rather than as a causal effect of the steroids per se. Hence, if women who received repeat steroids represented an intrinsically high risk group, the treatment may appear to be associated with worse outcomes (or a beneficial effect may be masked) through the effect of bias. Therefore, RCTs were essential to further evaluate this question.

Five RCTs have been published on this topic since 2001 (table 1). One study demonstrated no difference in functional residual capacity and respiratory compliance, ${ }^{19}$ but this study was small with only 37 patients randomised. In a larger study, Guinn et al did not find a significant difference in composite neonatal morbidity; however, in the subgroup with delivery between 24 and 27 weeks gestation, morbidity was reduced. ${ }^{20}$ In addition, severe RDS and severe IVH were both significantly less frequent in the group receiving repeated courses. An RCT from Finland reported increased respiratory morbidity, but its treatment group received only a single extra dose of betamethasone when delivery was imminent or electively planned within $48 \mathrm{~h}^{21}$ Recently two large RCTs, one from Australasia and one from the USA, both reported significantly reduced neonatal respiratory morbidity as demonstrated by less use of mechanical ventilation, oxygen therapy and surfactant. ${ }^{22}$ Patent ductus arteriosus and pulmonary air leak were also reduced in both studies, but this was of variable statistical significance. The incidence of other neonatal morbidities such as IVH, periventricular leukomalacia, BPD and retinopathy of prematurity was the same. Of note, the mean gestational age at delivery was 32.5 and 34.8 weeks in these studies and the high gestational age may mask potentially significant effects in more immature infants. For example, several neonatal morbidities including BPD and sepsis were less frequent in the repeat steroid group for deliveries at $<32$ weeks. ${ }^{22}$ A Cochrane review of these trials ${ }^{24}$ demonstrated that, overall, repeat glucocorticoids were associated with a significant reduction in the occurrence (relative risk: $0.82,95 \%$ CI 0.72 to 0.93 ) and severity (relative risk: 0.60 , $95 \%$ CI 0.48 to 0.75 ) of lung disease in neonatal life. Moreover, there was a significant reduction in a composite of severe morbidity (relative risk: 0.79, 95\% CI 0.67 to 0.93 )

\section{ADVERSE EFFECTS}

There are no strong data indicating a major concern regarding adverse maternal 